\title{
Transient Stability Analysis of Microgrids with a Line-based Model
}

\author{
Yue Song, David J. Hill and Tao Liu \\ Department of Electrical and Electronic Engineering \\ The University of Hong Kong \\ Hong Kong, China \\ Email: \{yuesong, dhill, taoliu\}@eee.hku.hk
}

\begin{abstract}
This paper investigates transient stability of microgrids with radial topology from the line-based perspective rather than the bus dynamics perspective. A line-based model is proposed to describe the dynamics of angle differences across transmission lines. Based on this model, the equilibrium points of the system are expressed in explicit form and the local stability is characterized. Then, a computationally efficient method to estimate the stability region is developed by using a topological Lyapunov function, where the critical energy for transient stability is evaluated by traversing the value of the topological Lyapunov function over a small and finite set of equilibrium points. This method also helps to identify the topological weakness in the system. Moreover, we build up easyto-solve optimization problems to measure the impact of power variations of renewables and loads on the stability level. The results are validated by numerical studies on a 9-bus test system.
\end{abstract}

Index Terms-microgrid, radial network, transient stability

\section{INTRODUCTION}

Thanks to the advances in renewable energy technology, power generation has been evolving from large generators to distributed generators (DGs), from which emerges the concept of a microgrid. Microgrids provide a way to integrate DGs, loads and control devices in low-voltage or medium-voltage power networks. They can operate autonomously either in integrated mode via the point of common coupling (PCC) or in islanded mode to support the critical loads [1]. Most of DGs, such as wind turbines and photovoltaic cells, need inverters as interfaces to be connected to microgrids. Due to the zero inertia of inverter-based DGs, the time constants of microgrids are much smaller than for conventional power systems with a large proportion of synchronous generators, which leads to less capacity for resisting disturbances. Thus more delicate analysis and control are required for the sake of stability.

In this paper, we focus on transient stability of microgrids, which refers to the ability to maintain angle synchronism when subjected to large disturbances [2]. Similar to the transient stability analysis of conventional power systems,

This work was supported by the Hong Kong PhD Fellowship (HKPF) Scheme and the Research Grants Council of the Hong Kong Special Administrative Region under the General Research Fund (GRF) through Project No. 17202414. transient stability problems of microgrids have also been recently studied by time domain simulation and the direct methods [3]. Transient stability of a microgrid in islanded mode with incidents triggered by faults was investigated in [4] in a simulation-based way. It reveals that the critical fault clearing time highly depends on microgrid control strategies and load types. Besides stability assessment, the data obtained by simulation is also useful for stability control. Trajectory sensitivity analysis was adopted in [5] to determine the site and size of capacitive compensator to improve the first swing stability of DGs. In [6], a controller was proposed to modify the frequency droop gain of an inverter by the information of frequency changing rate. The control effect serves as virtual inertia to eliminate frequency oscillation.

Direct methods provide an analytical viewpoint to transient stability, by which we can estimate the stability region so that stability can be checked without solving system dynamics numerically. In principle, the direct methods developed on conventional power systems can be applied to microgrids, such as the closest unstable equilibrium point (UEP) method and controlling UEP method (refer to [7] and references therein). Furthermore, as indicated in [8], inverter-based DGs can be modeled as Kuramoto oscillators. Hence, the methods for synchronization of the Kuramoto model have also been introduced into the study of microgrid dynamics. These methods view microgrid stability from the perspective of state agreement across transmission lines and lead to more concentration on the role of network topology. So far most literature [9-12] mainly focus on local stability of equilibrium points in the principal region, where angle differences across all transmission lines are required to be less than $90^{\circ}$. In [13], the local stability analysis is extended to the equilibrium points outside the principal region by using graph-theoretic methods. Nevertheless, the problem of state agreement across transmission lines under large disturbances, where nonlinear dynamics have to be included, still remains to be studied.

Along this direction, we propose a new model and derive some novel results on transient stability of microgrids in this paper. The main contributions are twofold: 1) For microgrids with radial network structure, angle differences across transmission lines are taken as state variables to build a new line-based model. With this model, equilibrium points are expressed explicitly and local stability is comprehensively 
characterized. 2) A method to fast estimate the stability region is proposed, the critical energy for transient stability is given by traversing the value of a topological Lyapunov function over a finite set of equilibrium points. And the line with the minimum energy storage is identified as the topological weakness. In addition, the impact of power variations on stability level is measured via easy-to-solve optimization problems.

The remainder of the paper is organized as follows. A linebased microgrid model is built up in Section II. Its equivalence to the bus dynamics-oriented model is proved. By the linebased model, equilibrium points are characterized in Section III. Besides, a fast estimation of the critical energy and stability region is developed, as well as a method to measure the impact of power variations. Section IV verifies the results on a 9-bus test system, some interesting phenomena raised are discussed. Section V concludes the paper.

\section{PROBLEM FORMULATION}

\section{A. Notations}

Let $\boldsymbol{A}$ be a matrix, $|\boldsymbol{A}|$ denotes the entry-wise absolute value of $\boldsymbol{A}$. For simplicity, a diagonal matrix $\boldsymbol{A} \in \mathbb{R}^{p \times p}$ is denoted as $\boldsymbol{A}=\operatorname{diag}\left\{a_{i}\right\}$ where $a_{i}$ is the $i$-th diagonal entry. A vector $\boldsymbol{x}=\left[x_{1}, \ldots, x_{p}\right]^{T} \in \mathbb{R}^{p}$ is denoted as $\boldsymbol{x}=\left[x_{i}\right] \in \mathbb{R}^{p}$ where $x_{i}$ is the $i$-th component. For $\boldsymbol{x}, \boldsymbol{y} \in \mathbb{R}^{p}$, the vector inequality $\boldsymbol{x}>\boldsymbol{y}$ represents the component-wise inequalities $x_{i}>y_{i}, i=1,2, \ldots, p$. Similar interpretation applies to other operators $\geq,<$ and $\leq$. Further, $f(\boldsymbol{x}) \in \mathbb{R}^{p}$ represents the function vector $f(\boldsymbol{x})=\left[f\left(x_{i}\right)\right] \in \mathbb{R}^{p}$, and $\mathbf{1}_{p} \in \mathbb{R}^{p}$ denotes the vector whose components are all equal to one.

\section{B. Line-based model of microgrid}

We begin with the microgrid model introduced in [11]. Consider a microgrid integrated with inverter-based DGs and frequency-dependent loads. The microgrid network is assumed lossless, which can be justified for medium voltage networks. The inverter-based DGs are modeled as ideal voltage sources with frequency droop controllers. Then, the dynamics of bus $i$ is described by

$$
\begin{aligned}
0= & P_{R_{i}}-D_{R_{i}} \dot{\theta}_{i}-\left(P_{L_{i}}+D_{L_{i}} \dot{\theta}_{i}\right) \\
& -\sum_{j \in \operatorname{adj}(i)} V_{i} V_{j}\left|Y_{i j}\right| \sin \left(\theta_{i}-\theta_{j}\right)
\end{aligned}
$$

where $P_{R_{i}}$ and $D_{R_{i}}$ are the nominal active power generation and the reciprocal of the frequency droop gain of the inverterbased DG at bus $i$, respectively, and $P_{L_{i}}$ and $D_{L_{i}}$ are the nominal load and the frequency coefficient of the load at bus $i$, respectively. The notation $j \in \operatorname{adj}(i)$ means there exists a transmission line connecting bus $i$ and bus $j$, where $\operatorname{adj}(i)=$ $\left\{j|| Y_{i j} \mid>0, j \neq i\right\}$ is the index set of the neighbouring buses for bus $i$ and $-Y_{i j}=-Y_{j i}$ is the admittance of the line $(i, j)$. Transformers are also regarded as lines. System (1) can represent microgrid dynamics either in islanded mode or integrated to the main grid. In the latter case, the PCC is regarded as an infinite bus with fictitious load that represents the power transfer between the microgrid and the main grid.
The fictitious load is positive when the power provided by DGs is in surplus and negative otherwise. Due to the existence of PCC, we have $\mathbf{1}_{n}^{T} \boldsymbol{P}=0$ for an integrated microgrid, while it is not necessarily true for an islanded microgrid.

Let $\mathcal{V}$ be the set of buses, and $\mathcal{E}$ be the set of transmission lines. The line $(i, j)$ is denoted as $e_{k}=(i, j) \in \mathcal{E}$ where $(i, j)$ is an unordered pair of buses. The cardinality of $\mathcal{V}$ and $\mathcal{E}$ are $n$ and $l$, respectively. Then, the microgrid network can be represented by the graph $\mathcal{G}(\mathcal{V}, \mathcal{E})$. We also introduce the incidence matrix $\boldsymbol{E} \in \mathbb{R}^{n \times l}$ of $\mathcal{G}(\mathcal{V}, \mathcal{E})$. To define it, we assign an arbitrary but fixed orientation for each line $e_{k} \in \mathcal{E}$, here, $e_{k}=(i, j)$ means the line $e_{k}$ starts at node $i$ and ends at node $j$. Accordingly $\boldsymbol{E}$ is defined as $E_{i k}=1, E_{j k}=-1$ and $E_{m k}=0, m \neq i, j, \forall e_{k}=(i, j) \in \mathcal{E}$. By using the incidence matrix, system (1) can be expressed as

$$
\boldsymbol{D} \dot{\boldsymbol{\theta}}=\boldsymbol{P}-\boldsymbol{E} \boldsymbol{B}_{l} \sin \left(\boldsymbol{E}^{T} \boldsymbol{\theta}\right)
$$

where $\boldsymbol{\theta}=\left[\theta_{i}\right] \in \mathbb{R}^{n}$ is the vector of bus angles, $\boldsymbol{D}=$ $\operatorname{diag}\left\{D_{i}\right\} \in \mathbb{R}^{n \times n}$ is the diagonal matrix of bus total damping coefficients where $D_{i}=D_{R_{i}}+D_{L_{i}}$, and $\boldsymbol{B}_{l}=\operatorname{diag}\left\{B_{l, k}\right\} \in$ $\mathbb{R}^{l \times l}$ is the diagonal matrix of line coupling strengths where $B_{l, k}=V_{i} V_{j}\left|Y_{i j}\right|, \forall e_{k}=(i, j) \in \mathcal{E}$. The vector $\boldsymbol{P}=\left[P_{i}\right] \in$ $\mathbb{R}^{n}$ is the bus injected power where $P_{i}=P_{R_{i}}-P_{L_{i}}, P_{i}>0$ means bus $i$ injects power to the network while $P_{i}<0$ means bus $i$ draws power from the network. Note that different line orientation assignments in defining $\boldsymbol{E}$ will not influence the expression of (2). Some assumptions are adopted in the sequel:

(A1) Voltage magnitude of each bus is constant.

(A2) The power network is connected and radial.

(A3) $\boldsymbol{P}$ is constant.

(A4) $\boldsymbol{D}$ is positive definite.

Assumption (A1) leads to the pure transient stability problem decoupled from voltage dynamics. Assumption (A2) refers to the typical structure for distribution networks. Radial topology gives that $l=n-1$ and $\boldsymbol{E}$ is an $n$ by $n-1$ matrix, which will be shown to make the problem more mathematically tractable. Assumption (A3) is commonly used in the stability analysis of transmission networks. The typical time scale of transient stability for conventional power systems is about 3 to 5 seconds [2], during which $\boldsymbol{P}$ keeps almost constant. Since the time scale for microgrids is even shorter due to zero rotor inertia, it is still reasonable to adopt (A3). Further, the nature of renewables and loads lead to quite high uncertainties of the bus injected power $\boldsymbol{P}$, how the stability level changes with different $\boldsymbol{P}$ is also of interest and will be detailed in Section III-C. Assumption (A4) reflects the common condition that frequency droop gains of inverters and frequency coefficients of loads are positive. In practice, there may exist bus $i$ with neither inverter-based DG nor frequency-dependent load so that $D_{i}=0$ and (A4) does not hold. For such cases, we can use singular perturbation to set $D_{i}$ as $D_{i}=\epsilon$ where $\epsilon$ is an arbitrary and small positive number. According to [14], the error between the original system and the singularly perturbed system will be sufficiently small if $\epsilon$ is sufficiently small. 
With these assumptions, we start to build the line-based model. Firstly, we implement the transform

$$
\theta_{i}^{\prime}=\theta_{i}-\frac{\mathbf{1}_{n}^{T} \boldsymbol{D} \boldsymbol{\theta}}{\mathbf{1}_{n}^{T} \boldsymbol{D} \mathbf{1}_{n}}
$$

to system (2) and it follows that

$$
\boldsymbol{D} \dot{\boldsymbol{\theta}}^{\prime}=\boldsymbol{P}^{\prime}-\boldsymbol{E} \boldsymbol{B}_{l} \sin \left(\boldsymbol{E}^{T} \boldsymbol{\theta}^{\prime}\right)
$$

where $\boldsymbol{P}^{\prime}=\boldsymbol{P}-\frac{\mathbf{1}_{n}^{T} \boldsymbol{P}}{\mathbf{1}_{n}^{T} \boldsymbol{D} \mathbf{1}_{n}} \boldsymbol{D} \mathbf{1}_{n}$ so that $\mathbf{1}_{n}^{T} \boldsymbol{P}^{\prime}=0$. Since system (4) takes the same form as (2), we drop the superscripts of $\boldsymbol{P}$ and assume $\mathbf{1}_{n}^{T} \boldsymbol{P}=0$ henceforth regardless of whether the microgrid is in integrated mode or not.

Then, we give the following lemma as a foundation for the line-based model.

Lemma 1: If $\mathbf{1}_{n}^{T} \boldsymbol{P}=0$, then for any connected network, the following statements hold

1) $\boldsymbol{P}$ can be expressed as $\boldsymbol{P}=\boldsymbol{E} \boldsymbol{B}_{l} \boldsymbol{P}_{l}$ where $\boldsymbol{P}_{l}=$ $\boldsymbol{B}_{l}^{-1} \boldsymbol{E}^{\dagger} \boldsymbol{P} \in \mathbb{R}^{l}$ and $\boldsymbol{E}^{\dagger}$ is the Moore-Penrose inverse of $\boldsymbol{E}$.

2) $\boldsymbol{P}_{l}$ is the solution with minimal 2-norm to the equation $\boldsymbol{P}=\boldsymbol{E} \boldsymbol{B}_{l} \boldsymbol{x}$. If the network is radial, $\boldsymbol{P}_{l}$ is unique.

Proof: 1) We claim that the equation $\boldsymbol{P}=\boldsymbol{E} \boldsymbol{x}$ has a solution. Let $\boldsymbol{F}=\left[\begin{array}{ll}\boldsymbol{E} & \boldsymbol{P}\end{array}\right]$, which can be expanded as

$$
\boldsymbol{F}=\left[\begin{array}{ll}
\boldsymbol{E}_{1} & P_{1} \\
\boldsymbol{E}_{r} & \boldsymbol{P}_{r}
\end{array}\right]=\left[\begin{array}{c}
-\mathbf{1}_{n-1}^{T} \\
\boldsymbol{I}
\end{array}\right]\left[\begin{array}{ll}
\boldsymbol{E}_{r} & \boldsymbol{P}_{r}
\end{array}\right]=\boldsymbol{T}\left[\begin{array}{ll}
\boldsymbol{E}_{r} & \boldsymbol{P}_{r}
\end{array}\right]
$$

where $P_{1}$ is the first component of $\boldsymbol{P}$, and $\boldsymbol{P}_{r} \in \mathbb{R}^{n-1}$ consists of the remaining $n-1$ components of $\boldsymbol{P}$. $\boldsymbol{E}_{1} \in \mathbb{R}^{1 \times l}$ is the first row of $\boldsymbol{E}$, and $\boldsymbol{E}_{r} \in \mathbb{R}^{(n-1) \times l}$ consists of the remaining $n-1$ rows of $\boldsymbol{E}$. Equation (5) holds by $\mathbf{1}_{n}^{T} \boldsymbol{E}=\mathbf{0}^{T}$ and $\mathbf{1}_{n}^{T} \boldsymbol{P}=0$. Since $\operatorname{rank}(\boldsymbol{E})=n-1$ [15] and $\boldsymbol{E}_{1}$ is a linear combination of the rows of $\boldsymbol{E}_{r}$, we have $\operatorname{rank}\left(\boldsymbol{E}_{r}\right)=n-1$ so that $\left[\begin{array}{ll}\boldsymbol{E}_{r} & \boldsymbol{P}_{r}\end{array}\right]$ has full row rank. Together with the $\boldsymbol{T}$ in (5) having full column $\operatorname{rank}$, we have $\operatorname{rank}(\boldsymbol{F})=\operatorname{rank}(\boldsymbol{E})=$ $n-1$ [16]. Thus the equation $\boldsymbol{P}=\boldsymbol{E} \boldsymbol{x}$ is consistent, and $\boldsymbol{P}_{l}=\boldsymbol{B}_{l}^{-1} \boldsymbol{E}^{\dagger} \boldsymbol{P}$ is a solution to the equation $\boldsymbol{P}=\boldsymbol{E} \boldsymbol{B}_{l} \boldsymbol{P}_{l}$.

2) The second statement follows directly from the properties of the Moore-Penrose inverse [17].

With Lemma 1, we are ready to present the line-based model, given by multiplying $\boldsymbol{E}^{T}$ to both sides of (2)

$$
\dot{\boldsymbol{\sigma}}=\boldsymbol{L}_{E}(\mathcal{G}) \boldsymbol{B}_{l}\left(\boldsymbol{P}_{l}-\sin \boldsymbol{\sigma}\right)
$$

where $\boldsymbol{\sigma}=\boldsymbol{E}^{T} \boldsymbol{\theta}=\left[\sigma_{k}\right] \in \mathbb{R}^{l}$ is the vector of angle differences across transmission lines and $\boldsymbol{L}_{E}(\mathcal{G})=\boldsymbol{E}^{T} \boldsymbol{D}^{-1} \boldsymbol{E}$ is a matrix reflecting the network topology. Note that $\boldsymbol{L}_{E}(\mathcal{G})$ is positive definite since $\boldsymbol{E}$ has full column rank. The following result validates the equivalence between (2) and (6).

Theorem 1: Suppose the phase angle of one bus is selected as the reference, e.g., bus 1 is the reference bus and $\theta_{1}$ is fixed to zero. Then for any radial network, the trajectory of (6), say $\boldsymbol{\sigma}(t)$, and the trajectory of (2), say $\boldsymbol{\theta}(t)$, are uniquely converted according to $\boldsymbol{\sigma}(t)=\boldsymbol{E}^{T} \boldsymbol{\theta}(t)$.

Proof: We show that $\left[\begin{array}{ll}\boldsymbol{E} & \boldsymbol{e}_{1}\end{array}\right]$ is nonsingular for radial networks, where $e_{1} \in \mathbb{R}^{n}$ with the first component being one and the others zero. If this is not true, then by $\operatorname{rank}(\boldsymbol{E})=n-1$, there must exist a nonzero $\boldsymbol{x} \in \mathbb{R}^{n-1}$ such that $\boldsymbol{e}_{1}=\boldsymbol{E} \boldsymbol{x}$. The last $n-1$ rows of this equation can be written as $\mathbf{0}=\boldsymbol{E}_{r} \boldsymbol{x}$. As mentioned before, $\operatorname{rank}\left(\boldsymbol{E}_{r}\right)=n-1$, then $\boldsymbol{E}_{r} \in \mathbb{R}^{(n-1) \times(n-1)}$ is nonsingular and we have $\boldsymbol{x}=\mathbf{0}$, which contradicts to that $\boldsymbol{x}$ is nonzero. Thus, the following equation

$$
\left[\begin{array}{c}
\boldsymbol{E}^{T} \\
\boldsymbol{e}_{1}^{T}
\end{array}\right] \boldsymbol{\theta}(t)=\left[\begin{array}{c}
\boldsymbol{\sigma}(t) \\
0
\end{array}\right]
$$

has a unique solution. This implies that a trajectory of (6) can be uniquely converted to a trajectory of (2) with $\theta_{1}=0$. Conversely, it is trivial to show that $\boldsymbol{\theta}(t)$ can be uniquely converted to $\boldsymbol{\sigma}(t)$ by $\boldsymbol{\sigma}(t)=\boldsymbol{E}^{T} \boldsymbol{\theta}(t)$.

We introduce the definitions of the principal region and transient stability, which show the merit of the line-based model.

Definition 1: [11] The principal region is defined as

$$
\mathcal{R}_{p}=\left\{\boldsymbol{\sigma} \mid-\frac{\pi}{2} \cdot \mathbf{1}_{l}<\boldsymbol{\sigma}<\frac{\pi}{2} \cdot \mathbf{1}_{l}\right\}
$$

Definition 2: Suppose the post-fault system is described by (6). Then transient stability is achieved if the trajectory of (6) approaches an equilibrium point in the principal region.

We can see that transient stability is more oriented to the dynamics of $\boldsymbol{\sigma}$ than $\boldsymbol{\theta}$. Thus, the line-based model would be more desirable for stability analysis. Furthermore, it can provide a topological view on stability since $\boldsymbol{\sigma}$ contains the information of network topology.

In the next section we will show that the line-based model helps to characterize equilibrium points and develop a fast method to estimate the stability region. In addition, the impact of power variations of renewables and loads can be conveniently analyzed by the "good" properties of this model.

\section{LINE-BASED ANALYSIS ON STABILITY AND THE IMPACT OF POWER VARIATIONS}

\section{A. Equilibrium point analysis}

We discuss the properties of equilibrium points of system (6), as fundamental for transient stability analysis. The equilibrium point is described by letting $\dot{\sigma}=\mathbf{0}$ in (6)

$$
\mathbf{0}=\boldsymbol{L}_{E}(\mathcal{G}) \boldsymbol{B}_{l}\left(\boldsymbol{P}_{l}-\sin \boldsymbol{\sigma}\right) .
$$

Since both $\boldsymbol{L}_{E}(\mathcal{G})$ and $\boldsymbol{B}_{l}$ are nonsingular, equation (9) is equivalent to $\boldsymbol{P}_{l}=\sin \boldsymbol{\sigma}$. Thus the set of equilibrium points, say $\mathcal{S}_{e}$, can be expressed in terms of $\boldsymbol{P}_{l}=\left[P_{l, k}\right] \in \mathbb{R}^{l}$ as follows:

1) If $\left|\boldsymbol{P}_{l}\right|<\mathbf{1}_{l}$, then $\mathcal{S}_{e}$ consists of those points $\boldsymbol{\sigma}=\left[\sigma_{k}\right] \in$ $\mathbb{R}^{l}$ that satisfy

$$
\sigma_{k} \bmod (2 \pi)=\sin ^{-1} P_{l, k} \text { or } \pi-\sin ^{-1} P_{l, k} .
$$

In this case there is a unique equilibrium point in the principal region and the other equilibrium points are outside. The equilibrium point in the principal region, say $\boldsymbol{\sigma}^{s}=\left[\sigma_{k}^{s}\right]$ where $\sigma_{k}^{s}=\sin ^{-1} P_{l, k}$, is the normal operating point. This operating point is locally asymptotically stable, while the equilibrium points with at least one line $e_{k} \in \mathcal{E}$ such that $\sigma_{k} \bmod (2 \pi)=\pi-\sin ^{-1} P_{l, k}$ 
are UEPs. The model in [18] with zero rotor inertia reduces to (6), so this result follows.

2) If $\left|\boldsymbol{P}_{l}\right| \leq \mathbf{1}_{l}$ and there exists a line $e_{k} \in \mathcal{E}$ such that $\left|P_{l, k}\right|=1$, then we have $\sigma_{k} \bmod (2 \pi)=\operatorname{sign}\left(P_{l, k}\right) \cdot \frac{\pi}{2}$ for those $e_{k}$ with $\left|P_{l, k}\right|=1$, where $\operatorname{sign}(\cdot)$ is the sign function. In this case no equilibrium point lies in the principal region.

3) If there exists a line $e_{k} \in \mathcal{E}$ such that $\left|P_{l, k}\right|>1$, then the equation $P_{l, k}=\sin \sigma_{k}$ has no solution and no equilibrium point exists for system (6).

Consider a 3-bus system as an example with the diagram and parameters in Fig. 1. The equilibrium points of the system are marked by the black dots in Fig. 2. We can see $\boldsymbol{\sigma}^{s}$ is located in the principal region bounded by the green dash line. The other equilibrium points are outside the principal region.

Remark 1: With the line-based model, an explicit form of equilibrium points is presented. The existence and stability of equilibrium points both inside and outside the principal region are elaborated. It can be concluded that there exists a unique stable equilibrium point in the principal region if and only if $\left|\boldsymbol{P}_{l}\right|<\mathbf{1}_{l}$. This criterion has analogs in the parallel literature on Kuramoto oscillators, see [19, Corollary 7.5]. The obtained results can be extended to more general cases. When reactive power flow equations are considered so that bus voltages become variables, the results still apply if the angle dynamics and voltage dynamics satisfy the assumption that they are highly decoupled [20]. Moreover, the points given by (10) can be regarded as an approximation of the equilibrium points for weakly meshed networks.

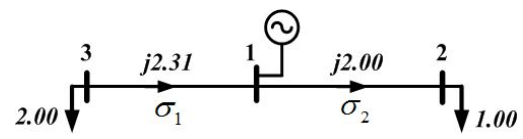

Figure 1. Diagram of a 3-bus system.

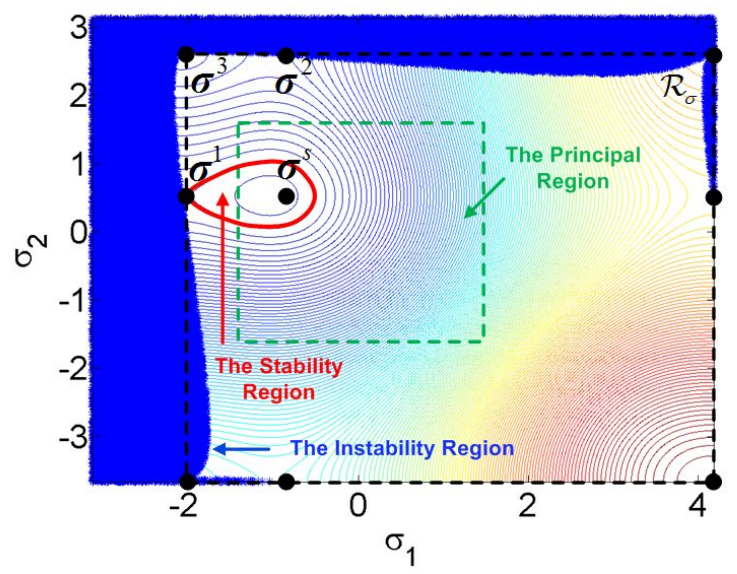

Figure 2. Profile of equilibrium points and energy contours.

\section{B. Transient stability analysis}

We proceed to transient stability analysis. We assume $\left|\boldsymbol{P}_{l}\right|<\mathbf{1}_{l}$ to guarantee the existence of the stable equilibrium point in the principal region, which is a necessary condition for transient stability. The stability of the equilibrium points outside the principal region is not concerned since they are inadequate for system operation even if they are stable. Hence, we focus on estimating the stability region of $\boldsymbol{\sigma}^{s}$ as follows.

Definition 3: [7] The stability region of the stable equilibrium point $\boldsymbol{\sigma}^{s}$, say $\mathcal{R}_{s}$, is the set that a trajectory $\boldsymbol{\sigma}(t)$ of system (6) with $\boldsymbol{\sigma}\left(t_{0}\right) \in \mathcal{R}_{s}$ will converge to $\boldsymbol{\sigma}^{s}$.

Similar to the case of conventional power systems, we estimate $\mathcal{R}_{s}$ using Lyapunov analysis. Firstly, we define a region $\mathcal{R}_{\sigma}$ as follows [21]

$$
\begin{aligned}
& \mathcal{R}_{\sigma}=\left\{\boldsymbol{\sigma} \mid \boldsymbol{\sigma}^{\min }<\boldsymbol{\sigma}<\boldsymbol{\sigma}^{\max }\right\} \\
& \sigma_{k}^{\max }=\pi-\sigma_{k}^{s} \\
& \sigma_{k}^{\min }=-\pi-\sigma_{k}^{s} .
\end{aligned}
$$

Let $\partial \mathcal{R}_{\sigma}$ be the boundary of $\mathcal{R}_{\sigma}$, where at least one component of $\boldsymbol{\sigma}$ takes its maximum or minimum value. From the discussion in Section III-A, we can conclude that there is a unique stable equilibrium point $\boldsymbol{\sigma}^{s}$ in $\mathcal{R}_{\sigma}$ and other $3^{l}-1$ UEPs in $\partial \mathcal{R}_{\sigma}$. As illustrated in Fig. $2, \mathcal{R}_{\sigma}$ is bounded by the black dash line. A unique stable equilibrium point $\sigma^{s}$ is located in $\mathcal{R}_{\sigma}$, while the other eight UEPs lie on $\partial \mathcal{R}_{\sigma}$.

Then, the following lemma establishes a Lyapunov function in $\mathcal{R}_{\sigma}$.

Lemma 2: Let $V(\boldsymbol{\sigma})=\mathbf{1}_{l}^{T} \boldsymbol{B}_{l}\left(\cos \boldsymbol{\sigma}^{s}-\cos \boldsymbol{\sigma}\right)+\boldsymbol{P}_{l}^{T} \boldsymbol{B}_{l}\left(\boldsymbol{\sigma}^{s}-\right.$ $\boldsymbol{\sigma})$. Then the following statements hold

1) $V(\boldsymbol{\sigma})$ is a Lyapunov function for the system (6) in $\mathcal{R}_{\sigma}$.

2) For any $\boldsymbol{\sigma}, \boldsymbol{\sigma}^{\prime} \in \mathcal{R}_{\sigma}$, we have $V\left(\boldsymbol{\sigma}^{\prime}\right) \geq V(\boldsymbol{\sigma})$ if $\sigma_{k}^{\prime}-$ $\sigma_{k}^{s} \geq \sigma_{k}-\sigma_{k}^{s} \geq 0$ or $\sigma_{k}^{\prime}-\sigma_{k}^{s} \leq \sigma_{k}-\sigma_{k}^{s} \leq 0, \forall k=$ $1,2, \ldots, l$.

Proof: 1) Let

$$
V_{k}\left(\sigma_{k}\right)=B_{l, k}\left(\cos \sigma_{k}^{s}-\cos \sigma_{k}+\sigma_{k}^{s} \sin \sigma_{k}^{s}-\sigma_{k} \sin \sigma_{k}^{s}\right)
$$

be the energy stored in the line $e_{k}$. It can be verified that $V(\boldsymbol{\sigma})=\sum_{e_{k} \in \mathcal{E}} V_{k}\left(\sigma_{k}\right)$. Consider that $V_{k}\left(\sigma_{k}^{s}\right)=0$ and $\frac{d V_{k}}{d \sigma_{k}}=$ $B_{l, k}\left(\sin \sigma_{k}-\sin \sigma_{k}^{s}\right)$, we have $\frac{d V_{k}}{d \sigma_{k}}>0, \sigma_{k} \in\left(\sigma_{k}^{s}, \sigma_{k}^{\max }\right)$ and $\frac{d V_{k}}{d \sigma_{k}}<0, \sigma_{k} \in\left(\sigma_{k}^{\min }, \sigma_{k}^{s}\right)$ so that $V_{k}\left(\sigma_{k}\right)>V_{k}\left(\sigma_{k}^{s}\right)=0$, $\forall \sigma_{k} \neq \sigma_{k}^{s}$. Thus $V\left(\boldsymbol{\sigma}^{s}\right)=0$ and $V(\boldsymbol{\sigma})>0, \forall \boldsymbol{\sigma} \in \mathcal{R}_{\sigma} \backslash\left\{\boldsymbol{\sigma}^{s}\right\}$.

Taking the time derivative of $V(\sigma)$ along the solution of the system (6) gives

$$
\dot{V}(\boldsymbol{\sigma})=-\left(\boldsymbol{P}_{l}-\sin \boldsymbol{\sigma}\right)^{T} \boldsymbol{B}_{l} \boldsymbol{L}_{E}(\mathcal{G}) \boldsymbol{B}_{l}\left(\boldsymbol{P}_{l}-\sin \boldsymbol{\sigma}\right) .
$$

Since $\boldsymbol{B}_{l} \boldsymbol{L}_{E}(\mathcal{G}) \boldsymbol{B}_{l}$ is positive definite, we have $\dot{V}(\boldsymbol{\sigma})<0$, $\forall \boldsymbol{\sigma} \in \mathcal{R}_{\sigma} \backslash\left\{\boldsymbol{\sigma}^{s}\right\}$. Therefore $V(\boldsymbol{\sigma})$ satisfies the condition to be a Lyapunov function in $\mathcal{R}_{\sigma}$.

2) From the previous analysis on $V_{k}$ we have $V_{k}\left(\sigma_{k}^{\prime}\right) \geq$ $V_{k}\left(\sigma_{k}\right)$ if $\sigma_{k}^{\prime}-\sigma_{k}^{s} \geq \sigma_{k}-\sigma_{k}^{s} \geq 0$ or $\sigma_{k}^{\prime}-\sigma_{k}^{s} \leq \sigma_{k}-\sigma_{k}^{s} \leq 0$. Thus the second statement holds.

The first statement of Lemma 2 validates $V(\boldsymbol{\sigma})$ as a Lyapunov function in $\mathcal{R}_{\sigma}$. The expression of $V(\boldsymbol{\sigma})$ is analogous to the potential energy of the topological Lyapunov function for power systems with synchronous generators [21], henceforth we also call it a topological Lyapunov function. Moreover, the second statement of Lemma 2 helps to develop a fast 
estimation of the stability region $\mathcal{R}_{s}$. To see this, we identify a specific set of equilibrium points on $\partial \mathcal{R}_{\sigma}$ as

$$
\begin{gathered}
\mathcal{S}_{c r i}=\left\{\boldsymbol{\sigma}^{m}=\left[\sigma_{k}^{m}\right], m=1,2, \ldots, l\right\} \\
\sigma_{k}^{m}= \begin{cases}\sigma_{k}^{\max }, & k=m, P_{l, k}>0 \\
\sigma_{k}^{\min }, & k=m, P_{l, k} \leq 0 \\
\sigma_{k}^{s}, & k \neq m\end{cases}
\end{gathered}
$$

and define the critical energy $V_{c r i}$ as

$$
V_{c r i}=\min _{\boldsymbol{\sigma}^{m} \in \mathcal{S}_{c r i}} V\left(\boldsymbol{\sigma}^{m}\right) \text {. }
$$

These definitions lead to the following results.

Theorem 2: Define the set $\Omega$ as

$$
\Omega=\left\{\boldsymbol{\sigma} \mid V(\boldsymbol{\sigma})<V_{\text {cri }}, \boldsymbol{\sigma} \in \mathcal{R}_{\sigma}\right\} .
$$

Then $\Omega \subseteq \mathcal{R}_{s}$, i.e., any trajectory of system (6) that starts from or enters into $\Omega$ will converge to $\boldsymbol{\sigma}^{s}$.

Proof: Let $V_{c r i}^{\prime}$ be the minimum value of $V(\boldsymbol{\sigma})$ along $\partial \mathcal{R}_{\sigma}:$

$$
V_{c r i}^{\prime}=\min _{\boldsymbol{\sigma} \in \partial \mathcal{R}_{\sigma}} V(\boldsymbol{\sigma}) .
$$

It can be concluded from the first statement of Lemma 2 that a trajectory of system (6) starting in or entering $\Omega^{\prime}=$ $\left\{\boldsymbol{\sigma} \mid V(\boldsymbol{\sigma})<V_{c r i}^{\prime}, \boldsymbol{\sigma} \in \mathcal{R}_{\sigma}\right\}$ will converge to $\boldsymbol{\sigma}^{s}$.

Then we show that $\forall \boldsymbol{\sigma}^{\prime} \in \partial \mathcal{R}_{\sigma} \backslash \mathcal{S}_{\text {cri }}$, there exists $\boldsymbol{\sigma}^{m} \in$ $\mathcal{S}_{c r i}$ that $V\left(\boldsymbol{\sigma}^{\prime}\right) \geq V\left(\boldsymbol{\sigma}^{m}\right)$ so that the optimization problem (15) is simplified into traversing over $\mathcal{S}_{c r i}$ and $V_{c r i}=V_{c r i}^{\prime}$. Let $\boldsymbol{\sigma}^{\prime} \in \partial \mathcal{R}_{\sigma} \backslash \mathcal{S}_{\text {cri }}$, and obviously at least one component of $\boldsymbol{\sigma}^{\prime}$ takes its extreme value. We suppose the $m$-th component takes its extreme value. Consider the case $\sigma_{m}^{\prime}=\sigma_{m}^{\max }=\pi-\sigma_{m}^{s}$, and compare $V\left(\boldsymbol{\sigma}^{\prime}\right)$ and $V\left(\boldsymbol{\sigma}^{m}\right)$ as follows:

1) If $P_{l, m}>0$ so that $\sigma_{m}^{m}=\sigma_{m}^{\max }=\pi-\sigma_{m}^{s}$ and $\sigma_{k}^{m}=\sigma_{k}^{s}$ for $k \neq m$, then $V_{m}\left(\sigma_{m}^{\prime}\right)=V_{m}\left(\sigma_{m}^{m}\right)$, and $\sigma_{k}^{\prime}-\sigma_{k}^{s} \geq \sigma_{k}^{m}-$ $\sigma_{k}^{s}=0$ or $\sigma_{k}^{\prime}-\sigma_{k}^{s} \leq \sigma_{k}^{m}-\sigma_{k}^{s}=0$ holds for $k \neq m$. By the second statement of Lemma 2, we have $V\left(\boldsymbol{\sigma}^{\prime}\right) \geq V\left(\boldsymbol{\sigma}^{m}\right)$.

2) If $P_{l, m} \leq 0$ so that $\sigma_{m}^{m}=\sigma_{m}^{\min }=-\pi-\sigma_{m}^{s}$ and $\sigma_{k}^{m}=\sigma_{k}^{s}$ for $k \neq m$, then it is trivial to check that $V_{m}\left(\sigma_{m}^{\prime}\right)>V_{m}\left(\sigma_{m}^{m}\right)$, and $\sigma_{k}^{\prime}-\sigma_{k}^{s} \geq \sigma_{k}^{m}-\sigma_{k}^{s}=0$ or $\sigma_{k}^{\prime}-\sigma_{k}^{s} \leq \sigma_{k}^{m}-\sigma_{k}^{s}=0$ holds for $k \neq m$. By the second statement of Lemma 2, we still have $V\left(\boldsymbol{\sigma}^{\prime}\right) \geq V\left(\boldsymbol{\sigma}^{m}\right)$.

The same result can be obtained when $\sigma_{m}^{\prime}=\sigma_{m}^{\min }$. It follows that $V_{c r i}=V_{c r i}^{\prime}$ and $\Omega=\Omega^{\prime}$. The proof is thus completed.

Remark 2: Theorem 2 gives an estimation of the stability region $\mathcal{R}_{s}$ by $\Omega$ that applies to all kinds of faults. The computational cost is low as $V_{\text {cri }}$ is evaluated just by traversing the value of $V(\boldsymbol{\sigma})$ over the finite set $\mathcal{S}_{c r i}$ whose elements are explicitly expressed. It avoids the time-consuming iterative process for finding the closest UEP that is needed in the conventional analysis. For example, in Fig. 2, we have $\mathcal{S}_{\text {cri }}=\left\{\boldsymbol{\sigma}^{1}, \boldsymbol{\sigma}^{2}\right\}$. By the contours of $V(\boldsymbol{\sigma})$ we can see that $V_{c r i}$ is reached by $\sigma^{1}$, then the estimated stability region bounded by the red line is quickly obtained. The blue area in Fig. 2 is part of the instability region starting from which the system trajectory will not converge to $\sigma^{s}$ (suppose $D_{1}=5.0, D_{2}=1.0, D_{3}=1.0$ ). It shows that the points in $\mathcal{R}_{\sigma}$ are very likely to be stable, which has similarities with the conjectures in [22] about the distribution of the attraction region of stable equilibrium point in radial networks. Moreover, it can be seen that there exist some points $\sigma$ in the instability region such that $V(\boldsymbol{\sigma})$ is just a little greater than $V_{\text {cri }}$. This justifies our selection to take $V\left(\boldsymbol{\sigma}^{1}\right)$ as $V_{\text {cri }}$ because the region surrounded by a larger threshold is not reliable at all. However, the set $\Omega$ fails to cover all the nonblue area, illustrating the inevitable conservativeness of the Lyapunov method. To give a broader estimation of the stability region, some techniques in the literature could be adopted. For instance, a more detailed estimation of the stability region in the principal region is given in [23], and an optimization model is proposed in [24] to seek the optimal paramters of the Lyapunov function for estimation enlargement.

Further, Theorem 2 also provides a useful topological perspective to the transient stability problem. Substituting the element of $\mathcal{S}_{\text {cri }}$ into $V(\boldsymbol{\sigma})$ gives

$$
\begin{aligned}
V\left(\boldsymbol{\sigma}^{m}\right) & =2 B_{l, m} \cos \sigma_{m}^{s}-B_{l, m}\left(\pi-2\left|\sigma_{m}^{s}\right|\right) \sin \left|\sigma_{m}^{s}\right| \\
m & =1,2, \ldots, l
\end{aligned}
$$

so that $V\left(\boldsymbol{\sigma}^{m}\right)$ is the energy stored in the line $e_{m}$. This implies that $V_{c r i}$ is decided by the line with the minimum energy storage, which coincides with the fact that two sub-networks are prone to be split at the line with small admittance and large power transfer. Therefore we regard this line as the topological weakness in the system. Taking the derivative of $V\left(\boldsymbol{\sigma}^{m}\right)$ gives

$$
\frac{\partial V\left(\boldsymbol{\sigma}^{m}\right)}{\partial \sigma_{m}^{s}}=-\operatorname{sign}\left(\sigma_{m}^{s}\right) B_{l, m}\left(\pi-2\left|\sigma_{m}^{s}\right|\right), \sigma_{m}^{s} \neq 0 .
$$

Since $\frac{\partial V\left(\boldsymbol{\sigma}^{m}\right)}{\partial \sigma_{m}^{s}}<0, \sigma_{m}^{s}>0$ and $\frac{\partial V\left(\boldsymbol{\sigma}^{m}\right)}{\partial \sigma_{m}^{s}}>0, \sigma_{m}^{s}<0, V_{\text {cri }}$ will be increased if we achieve smaller absolute values of angle differences across lines, especially for those lines with less energy storage that mainly determine $V_{c r i}$. This gives a direction for demand side management to improve stability.

\section{The impact of power variations}

Section III-B is concerned with the critical energy and stability region of a specific system snapshot, i.e., for a specific $\boldsymbol{P}$. Here we analyze how the variations of $\boldsymbol{P}$ will affect the stability level. Suppose the load and generation forecasts give that $\boldsymbol{P}$ is upper bounded by $\boldsymbol{P}^{\mathrm{max}}$ and lower bounded by $\boldsymbol{P}^{\mathrm{min}}$. Then we can formulate optimization problem to find the worst critical energy, say $V_{c r i}^{\mathrm{min}}$, within such interval. For a microgrid in integrated mode, the optimization problem can be formulated as (suppose bus 1 is the PCC)

$$
\begin{aligned}
V_{c r i}^{\min }=\min _{\boldsymbol{P}} & V_{c r i}\left(\boldsymbol{\sigma}^{s}\right) \\
\text { s.t. } & \boldsymbol{\sigma}^{s}=\sin ^{-1} \boldsymbol{B}_{l}^{-1} \boldsymbol{E}^{\dagger} \boldsymbol{P} \\
& \boldsymbol{P}=\boldsymbol{T} \boldsymbol{P}_{r}, \\
& \boldsymbol{P}_{r}^{\min } \leq \boldsymbol{P}_{r} \leq \boldsymbol{P}_{r}^{\max }
\end{aligned}
$$

where $V_{c r i}$ is written as $V_{c r i}\left(\boldsymbol{\sigma}^{s}\right)$ since we can see from (15) and (18) that $V_{c r i}$ is decided by $\boldsymbol{\sigma}^{s} . \boldsymbol{P}_{r}$ and $\boldsymbol{T}$ are defined in (5), $\boldsymbol{P}_{r}^{\min }$ and $\boldsymbol{P}_{r}^{\max }$ are the upper bound and lower bound of the injected power of the last $n-1$ buses. $\boldsymbol{P}=\boldsymbol{T} \boldsymbol{P}_{r}$ 
gives that $P_{1}=-\mathbf{1}_{n-1} \boldsymbol{P}_{r}$, i.e., the power deficit is balanced by the power transfer via PCC. The optimization problem for islanded microgrid is

$$
\begin{aligned}
& V_{c r i}^{\min }=\min _{\boldsymbol{P}} V_{c r i}\left(\boldsymbol{\sigma}^{s}\right) \\
& \text { s.t. } \boldsymbol{\sigma}^{s}=\sin ^{-1} \boldsymbol{B}_{l}^{-1} \boldsymbol{E}^{\dagger}\left(\boldsymbol{P}-\frac{\mathbf{1}_{n}^{T} \boldsymbol{P}}{\mathbf{1}_{n}^{T} \boldsymbol{D} \mathbf{1}_{n}} \boldsymbol{D} \mathbf{1}_{n}\right) \\
& \boldsymbol{P}^{\min } \leq \boldsymbol{P} \leq \boldsymbol{P}^{\max }
\end{aligned}
$$

where coordinate transform (3) is needed. Optimization problems (20) and (21) are easy to solve given the explicit form of $\boldsymbol{\sigma}^{s}$. In addition, problems (20) and (21) implicitly assume that $\left|\boldsymbol{B}_{l}^{-1} \boldsymbol{E}^{\dagger} \boldsymbol{P}\right|<\mathbf{1}_{l}$ holds for any $\boldsymbol{P}^{\mathrm{min}} \leq \boldsymbol{P} \leq \boldsymbol{P}^{\mathrm{max}}$, i.e., the existence of $\sigma^{s}$ is robust to power variations. We refer to Theorem 4 in the supporting information of [10] for details.

Denote the control variable corresponding to $V_{c r i}^{\min }$ as $\boldsymbol{P}^{*}=$ $\left[P_{i}^{*}\right] \in \mathbb{R}^{n}$ (suppose the $\boldsymbol{P}^{*}$ in $(21)$ is transformed so that $\left.\mathbf{1}_{n}^{T} \boldsymbol{P}^{*}=0\right)$. Then the power variation at bus $i$

$$
\max _{i}\left|\frac{P_{i}^{*}}{P_{i}}-1\right|, i=1,2, \ldots, n
$$

has the largest impact on the critical energy.

\section{CASE STUDY}

We study the 9-bus radial network shown in Fig. 3, where the bus and line parameters are in per unit value. Suppose all bus voltages are 1.0 p.u., and bus 1 is the PCC and a bus with DG in integrated mode and islanded mode, respectively. Simple calculation leads to that $V_{c r i}=9.0752$ and the line $(5,6)$ is identified as the topological weakness as $V_{c r i}$ is decided by its energy storage. This result can be interpreted from two aspects. Firstly, the line $(5,6)$ has the smallest admittance so that it is clearly vulnerable. Secondly, the line $(5,6)$ is the unique path to transmit power from generators to supply the load demand at bus 5 , which results in a large angle difference across it. By comparison, the load at bus 7 is supplied via the line $(7,8)$, which has much larger admittance. The load at bus 9 is supplied via the line $(9,4)$ and the line $(8,9)$ so that the power flow across each line is lighter. Thus the line $(5,6)$ is the weakest link.

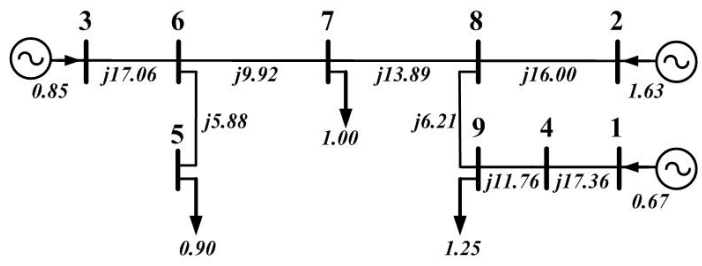

Figure 3. Diagram of the 9-bus test system.

Some strategies can be adopted to increase $V_{\text {cri }}$, such as load reduction and line capacity expansion. The former one can be achieved by demand side management while the latter one is about network expansion planning. For instance, if the load at bus 5 is reduced to 0.8 p.u., $V_{\text {cri }}$ will be increased to 9.3604 in integrated mode and 9.3445 in islanded mode. If the capacity of the line $(5,6)$ is doubled, $V_{c r i}$ will be increased to
10.6544 both in integrated mode and islanded mode, and the line $(8,9)$ becomes the weakest link so that the vulnerability of the line $(5,6)$ is significantly mitigated.

Next we analyze the impact of power variations on $V_{c r i}$. Suppose the generations and loads have $\pm 5 \%$ variation, then we get $V_{c r i}^{\min }=8.9480$ in integrated mode and $V_{c r i}^{\min }=8.9128$ in islanded mode, both are a bit lower than $V_{c r i}=9.0752$ with no variation. The $P_{i}^{*} / P_{i}$ data in these two modes are listed in Table I. For islanded mode, we adopt the transformed data in the last column as it is directly linked to equilibrium point. The load variation at bus 5 is identified to have the largest impact both in integrated and islanded mode, it reaches its upper limit to minimize the energy storage of the line $(5,6)$. Thus reducing variation at bus 5 will be the most effective way to improve the robustness of $V_{c r i}$. As a validation, suppose the variation at bus 5 is reduced to $\pm 2 \%$ while the variations at other buses are kept $\pm 5 \%$, then we have $V_{c r i}^{\min }=9.0243$ in integrated mode and $V_{c r i}^{\min }=8.9847$ in islanded mode, so that a significant increase is achieved. By comparison, if the variation at bus 7 or bus 9 is reduced to $\pm 2 \%, V_{c r i}^{\min }$ gets no change in integrated mode and only a slight increment in islanded mode.

The curve of $V_{c r i}^{\mathrm{min}}$ versus power variation is shown in Fig. 4. $V_{c r i}^{\min }$ declines almost linearly with the increasing variation degree. The green curve (integrated mode) is more flat than the blue curve (islanded mode) when the variation is less than $\pm 16 \%$. This makes sense since PCC is flexible to balance power to keep the system in a better status. A phenomenon of interest is that the green curve encounters a switching point when the variation reaches $\pm 16 \%$, afterwards the line $(8,9)$ replaces the line $(5,6)$ to be the weakest link. We list the $P_{i}^{*} / P_{i}$ data when the variation is $\pm 15 \%$ or $\pm 16 \%$ in Table II. The case with $\pm 15 \%$ variation is similar to the one with $\pm 5 \%$ variation in Table I, the load variation at bus 5 has the largest impact. However, in the case with $\pm 16 \%$ variation, $V_{c r i}^{\min }$ is caused by more global factors as both generators and loads make a contribution. We note that the large generations at bus 2 and bus 3 and light loads at bus 5 and bus 7 make the power transfer via PCC reversed. Since the line $(8,9)$ is the only path to transmit the surplus power to PCC, the power flow across it becomes so heavy that its energy storage becomes the minimum. Such global effect leads to the undesirable sharp drop on the green curve after the switching point.

The above discussion indicates that power variations may have a significant impact on the stability level, such as the switching point in Fig. 4 caused by reversed power transfer that makes the critical energy evaluation much less robust. Variation reduction strategies, such as more precise forecast and delicate control on renewables and loads, are needed especially for the bus identified to be the most responsible for the decline of critical energy.

\section{CONCLUSION}

Transient stability of radial microgrids has been studied on a new line-based model. The equilibrium points can be expressed in explicit form in this model, by which a fast 
TABLE I. THE WORST CRITICAL ENERGY WITH $\pm 5 \%$ VARIATION

\begin{tabular}{|c|c|c|c|}
\hline Bus & $P_{i}^{*} / P_{i}$ (integrated) & $P_{i}^{*} / P_{i}$ (islanded) & $P_{i}^{*} / P_{i}$ (islanded) \\
\hline 1 & 1.0614 (PCC) & 1.0500 & 1.0034 \\
2 & 1.0035 & 1.0500 & 1.0308 \\
3 & 1.0010 & 1.0500 & 1.0132 \\
4 & NA & NA & NA \\
5 & 1.0500 & 1.0500 & 1.0639 \\
6 & NA & NA & NA \\
7 & 1.0028 & 0.9500 & 0.9625 \\
8 & NA & NA & NA \\
9 & 0.9998 & 0.9500 & 0.9600 \\
\hline
\end{tabular}

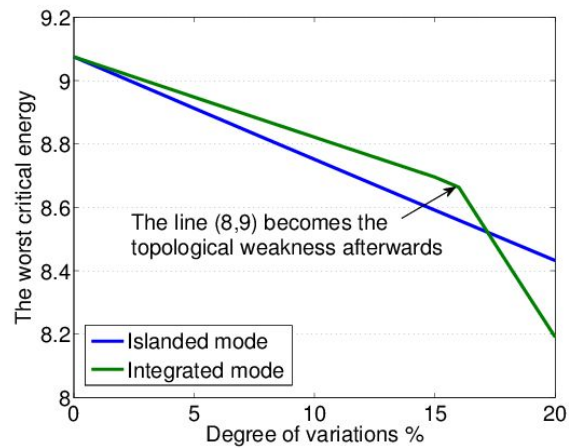

Figure 4. Impact of power variations on the worst critical energy.

TABLE II. THE WORST CRITICAL ENERGY IN INTEGRATED MODE WITH $\pm 15 \%$ OR $\pm 16 \%$ VARIATION

\begin{tabular}{|c|c|c|}
\hline Bus & $P_{i}^{*} / P_{i}( \pm 15 \%$ variation $)$ & $P_{i}^{*} / P_{i}( \pm 16 \%$ variation $)$ \\
\hline 1 & $1.1530(\mathrm{PCC})$ & $-0.0478(\mathrm{PCC})$ \\
2 & 1.0124 & 1.1600 \\
3 & 1.0000 & 1.1600 \\
4 & 0.0000 & 0.0000 \\
5 & 1.1500 & 0.8400 \\
6 & 0.0000 & 0.0000 \\
7 & 0.9944 & 0.8400 \\
8 & 0.0000 & 0.0000 \\
9 & 0.9947 & 0.9990 \\
\hline
\end{tabular}

estimation of the stability region is established. The critical energy for transient stability is obtained by traversing the value of the proposed topological Lyapunov function over a small and finite set of equilibrium points. This model also gives a topological view on transient stability, showing that system weakness is related to those lines with less energy storage, i.e., small admittance and heavy loaded lines. Furthermore, we propose two optimization problems to measure the impact of power variations of renewables and loads on transient stability level for integrated mode and islanded mode, respectively. The results have been validated by a 9-bus test system. It shows that power variations of renewables and loads can significantly impact the critical energy, and such impacts can be effectively mitigated by reducing power variation at the bus that is identified to bear the most responsibility. These conclusions lead to helpful suggestions for microgrid operation.

\section{REFERENCES}

[1] R. H. Lasseter, "Microgrids," in Power Engineering Society Winter Meeting, 2002. IEEE, vol. 1. IEEE, 2002, pp. 305-308.

[2] P. Kundur, J. Paserba, V. Ajjarapu, G. Andersson, A. Bose, C. Canizares, N. Hatziargyriou, D. Hill, A. Stankovic, C. Taylor, T. Van Cutsem, and V. Vittal, "Definition and classification of power system stability IEEE/CIGRE joint task force on stability terms and definitions," Power Systems, IEEE Transactions on, vol. 19, no. 3, pp. 1387-1401, Aug 2004.

[3] R. Majumder, "Some aspects of stability in microgrids," Power Systems, IEEE Transactions on, vol. 28, no. 3, pp. 3243-3252, Aug 2013.

[4] A. Kasem Alaboudy, H. Zeineldin, and J. L. Kirtley, "Microgrid stability characterization subsequent to fault-triggered islanding incidents," Power Delivery, IEEE Transactions on, vol. 27, no. 2, pp. 658-669, April 2012.

[5] A. Bidram, M.-e. Hamedani-golshan, and A. Davoudi, "Capacitor design considering first swing stability of distributed generations," Power Systems, IEEE Transactions on, vol. 27, no. 4, pp. 1941-1948, Nov 2012.

[6] N. Soni, S. Doolla, and M. C. Chandorkar, "Improvement of transient response in microgrids using virtual inertia," Power Delivery, IEEE Transactions on, vol. 28, no. 3, pp. 1830-1838, July 2013.

[7] H.-D. Chiang, C.-C. Chu, and G. Cauley, "Direct stability analysis of electric power systems using energy functions: theory, applications, and perspective," Proceedings of the IEEE, vol. 83, no. 11, pp. 1497-1529, Nov 1995

[8] J. W. Simpson-Porco, F. Dörfler, and F. Bullo, "Droop-controlled inverters in microgrids are kuramoto oscillators," in IFAC Workshop on Distributed Estimation and Control in Networked Systems, Santa Barbara, California, USA. Citeseer, 2012, pp. 264-269.

[9] J. W. Simpson-Porco, F. Dörfler, and F. Bullo, "Synchronization and power sharing for droop-controlled inverters in islanded microgrids," Automatica, vol. 49, no. 9, pp. 2603-2611, 2013.

[10] F. Dörfler, M. Chertkov, and F. Bullo, "Synchronization in complex oscillator networks and smart grids," Proceedings of the National Academy of Sciences, vol. 110, no. 6, pp. 2005-2010, 2013.

[11] N. Ainsworth and S. Grijalva, "A structure-preserving model and sufficient condition for frequency synchronization of lossless droop inverterbased ac networks," Power Systems, IEEE Transactions on, vol. 28, no. 4, pp. 4310-4319, Nov 2013 .

[12] J. Schiffer, R. Ortega, A. Astolfi, J. Raisch, and T. Sezi, "Conditions for stability of droop-controlled inverter-based microgrids," Automatica, vol. 50, no. 10, pp. 2457-2469, 2014.

[13] Y. Song, D. J. Hill, and T. Liu, "Small-disturbance angle stability analysis of microgrids: A graph theory viewpoint," in Control Applications (CCA), 2015 IEEE Conference on. IEEE, 2015, pp. 201-206.

[14] H. K. Khalil and J. Grizzle, Nonlinear systems. Prentice hall New Jersey, 1996, vol. 3.

[15] R. B. Bapat, Graphs and matrices. Springer, 2010.

[16] R. A. Horn and C. R. Johnson, Matrix analysis. Cambridge university press, 2012.

[17] A. Ben-Israel and T. N. Greville, Generalized inverses: theory and applications. Springer Science \& Business Media, 2003, vol. 15.

[18] D. J. Hill and A. R. Bergen, "Stability analysis of multimachine power networks with linear frequency dependent loads," Circuits and Systems, IEEE Transactions on, vol. 29, no. 12, pp. 840-848, Dec 1982

[19] F. Dörfler and F. Bullo, "Synchronization in complex networks of phase oscillators: A survey," Automatica, vol. 50, no. 6, pp. 1539-1564, 2014.

[20] J. W. Simpson-Porco, F. Dörfler, and F. Bullo, "A solvability condition for reactive power flow," in Decision and Control (CDC), 2015 IEEE 54th Annual Conference on, Dec 2015, pp. 2013-2017.

[21] A. R. Bergen and D. J. Hill, "A structure preserving model for power system stability analysis," Power Apparatus and Systems, IEEE Transactions on, vol. PAS-100, no. 1, pp. 25-35, Jan 1981.

[22] F. Dörfler and J. M. Hendrickx, "Synchronization of oscillators: Feasibility and non-local analysis," 2014.

[23] S. Backhaus, R. Bent, D. Bienstock, M. Chertkov, and D. Krishnamurthy, "Efficient synchronization stability metrics for fault clearing," arXiv preprint arXiv:1409.4451, 2014.

[24] T. Vu and K. Turitsyn, "Lyapunov functions family approach to transient stability assessment," Power Systems, IEEE Transactions on, vol. 31, no. 2, pp. 1269-1277, March 2016. 